\title{
The combined use of SEM, EPMA and FIB for the characterization of novel biomaterials for bone regeneration
}

\author{
Mouad Essani ${ }^{1}$, Patricia Abellan ${ }^{2}$, Pierre Weiss $^{3}$, Jean Le Bideau ${ }^{1}$, Baptiste Charbonnier ${ }^{4}$ and Hilel Moussi ${ }^{4}$ \\ ${ }^{1}$ Université de Nantes, CNRS, Institut des Matériaux Jean Rouxel (IMN), Nantes, Pays de la Loire, France, \\ ${ }^{2}$ IMN / University of Nantes, Nantes, France, ${ }^{3}$ Université de Nantes, INSERM U1229, Regenerative \\ Medicine and skeleton Research Lab (RMES), Nantes, Pays de la Loire, France, ${ }^{4}$ Université de Nantes, \\ INSERM U1229, Regenerative Medicine and skeleton Research Lab (RMES), United States
}

Bone has the innate ability to spontaneously heal without scarring through complex physiological processes [1]. Unfortunately, this regenerative ability is limited, and surgical interventions including the filling of bone defect are often required to achieve healing [2]. One of the therapeutic strategies used for bone regeneration consists on the injection of biomaterials embedded with bone marrow mesenchymal stem cells onto bone defect. Most widely used biomaterials include calcium phosphate (CaP) injectable bone substitutes (IBS) serving as fillers that will be replaced by a new bone. Despite significant advantages over other bone substitutes, IBS still lacks of suitable biodegradation rate and bioactivity which is partly due to the absence of an appropriate macroporous interconnected network and the presence of sintered CaP particles. Recent developments at Regenerative Medicine and skeleton Research (RMES) laboratory allowed for the elaboration of macroporous IBS based on reactive $\mathrm{CaP}$ powders and hydrogel matrix $[2,3]$. Biological in vivo evaluation showed that the IBS biodegradation and the regeneration of a critical-sized defect depended on the nature of the hydrogel and the inorganic phase as well as macroarchitecture of the implanted IBS. Correlating the biological response to the chemical and structural parameters of the biomaterial would allow for unveiling new prospects for the design of clinically relevant biomaterials for bone reconstruction. On the other hand, the characterization of the interactions between the biomaterial and the stem cells or between the host and biomaterial will provide insight into parameters impacting the biomaterial's biological performances.

Among advanced tools that were reported as efficient for chemical and structural analysis of materials exhibiting complex structures include the combined use of focused ion beam (FIB) and scanning electron microscopy (SEM) [4]. The analysis consists on serial sectioning using FIB and imaging with SEM which enables obtaining a 3D representation of the material microstructure [4,5]. Other studies showed the advantages of combining FIB, SEM and electron probe microanalysis (EPMA) for morphological and chemical characterization of samples [6]. These techniques can be substantially used to characterize both the hierarchical structure and chemical properties of IBS. Nevertheless, attention must be paid when applying such techniques to beam sensitive samples such as biomaterials. In addition, the high concentrations of light elements in these samples might lead to inaccurate chemical analysis with EPMA.

In this work, we present our latest results of analysis with FIB, SEM and EPMA on reference biomaterials (i.e. IBS before implantation). A first analysis was performed on a CaP - IBS mixed with gelatin [7], showing a highly porous structure (Fig 1). In order, to avoid degradation of samples under irradiation, analyses were performed with a low dose. Different tests were carried out in order to obtain optimal parameters for analyses with FIB. Serial sectioning of the region of interest in the sample was further performed with FIB along with imaging by SEM and energy dispersive spectroscopy (EDS) mapping. This enables obtaining a 3D representation of both microstructure and elemental distribution inside the mixture 
(Fig 2) which can provide a clear view on 3D pore morphology, pores connectivity inside the sample as well as internal diffusion of all elements.

Accurate 3D representation of both microstructure and elemental distribution can be obtained with the combined use of FIB, SEM and EDS. Analyses will be performed in cryogenic condition, which, in addition of protecting the biological samples, may allow for the observation of cell/biomaterial interactions (e.g., cell conformation, organization and composition of extracellular matrix). This can give insight into the biocompatibility and bioactivity of biomaterials used for bone regeneration.
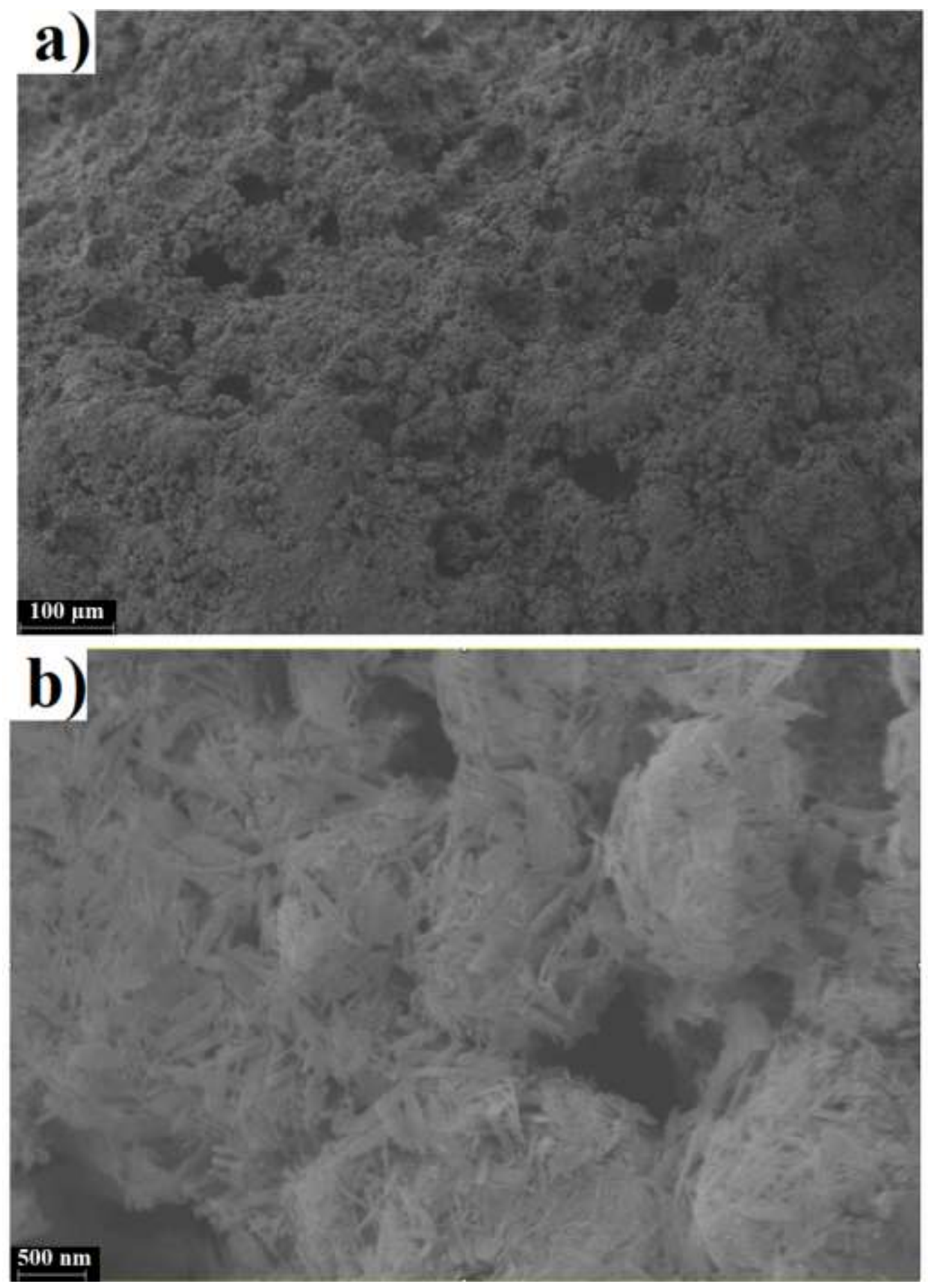
Figure 1. SEM images of cement/ gelatin mixture exhibiting highly porous complex structure with a) pores having a size of hundreds of micrometers and $b$ ) pores with a size ranging from tens to hundreds of nanometers.
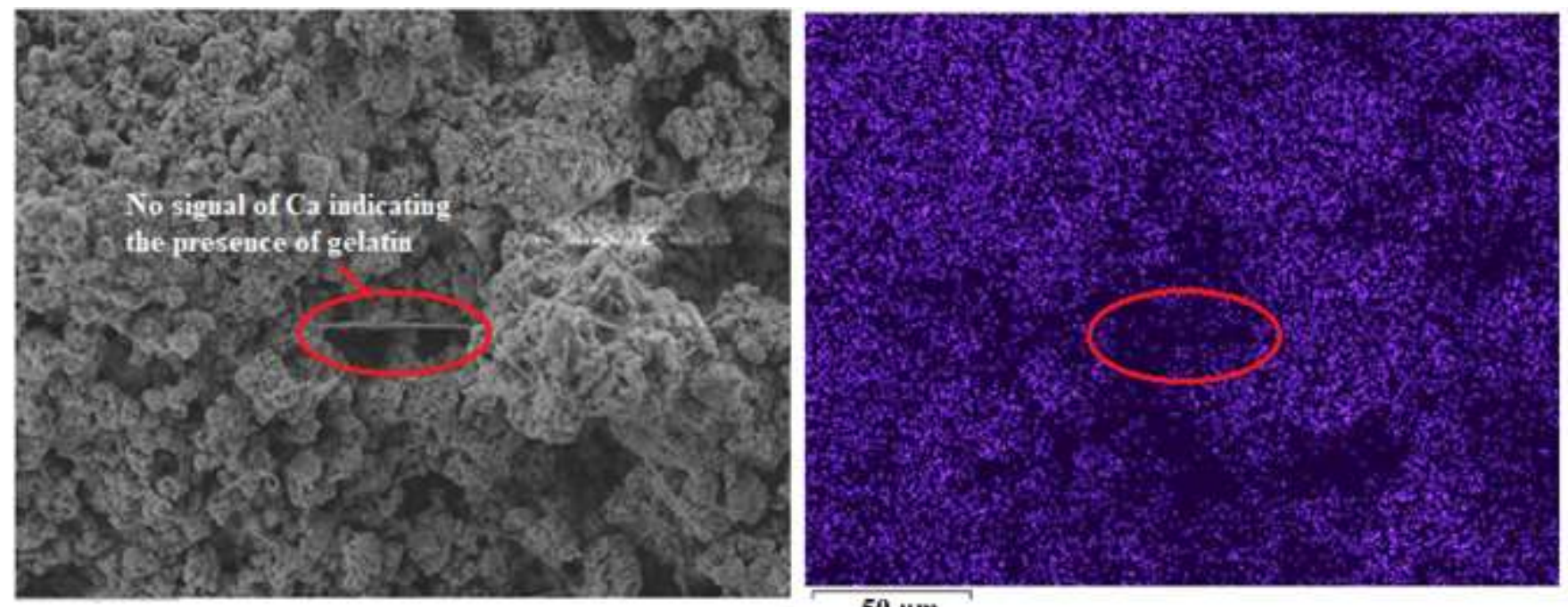

$50 \mu \mathrm{m}$

Figure 2. EDS mapping of calcium showing the presence of both CaP IBS and gelatin in the mixture.

\section{References}

[1] A. Oryana, S. Monazzah, A. Bigham-Sadeghc (2015). Biomed Environ Sci, Vol 28, pp. 57-71.

[2] I. Lodoso-Torrecilla, J.J.J.P Van den Beucken, J.A Jansen (2021). Acta Biomaterialia, 119, pp 1-12.

[3] H. Moussi et al (2021). To be published soon.

[4] M.A. Groeber, B.K. Haley, M.D. Uchic, D.M. Dimiduk, S. Ghosh (2006). Materials Characterization, 57 , pp. 259-273.

[5] B. Titze and C. Genoud (2016). Biol. Cell, 108, 307-323.

[6] M. Taheri, R. Phillips, J. Kish, G. Botton (2012), Corros. Sci., 59, pp. 222-228.

[7] N. Eliaz, N. Metoki (2017). Materials, 10 (4), 334. 